\section{Titanium alloy removable partial denture framework in a patient with a metal allergy: a case study}

\author{
F. Oluwajana ${ }^{1}$ and A. D. Walmsley²
}
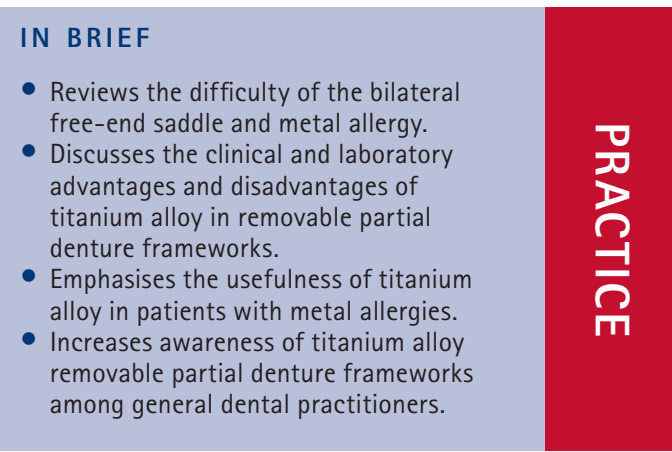

\author{
VERIFIABLE CPD PAPER
}

This article describes a mandibular bilateral free-end saddle case in a 63-year-old female with a metal allergy. Conventional denture alloys are contraindicated and acrylic mucosa-borne dentures were not tolerated. The use of a titanium alloy framework is shown to be a successful alternative in this case.

\section{CASE REPORT}

The patient presented with a Kennedy Classification 1 partially dentate mandibular arch and a distal extension removable partial denture (RPD). Her complaint was of looseness, instability and pain. The patient's existing acrylic mucosa-borne denture had wrought gold occlusally approaching clasps on the abutment teeth and lacked retention, support and stability.

At 63 years of age the patient had controlled hypertension, was a long-term smoker and reported a history of metal allergy. Patch testing revealed allergy to nickel, cobalt chloride, palladium chloride and gold sodium thiosulphate. The remaining mandibular dentition comprised of LR4, LR3, LR2, LR1 and LL1, LL2, LL3; the maxillary arch was edentulous and the upper prosthesis was being worn successfully. Clinically, the edentulous free-end saddles had undergone marked bone resorption with the bony anatomical features becoming prominent. The periodontal status of the remaining dentition was poor, with the central incisors being grade 3 mobile. Lateral spread of the tongue had occurred due to loss of the posterior dentition.

\footnotetext{
${ }^{1 *}$ Fourth year BDS student, ${ }^{2}$ Professor of Restorative Dentistry, School of Dentistry, St Chad's Queensway, Birmingham, B4 6NN:

${ }^{*}$ Correspondence to: Funmi Oluwajana

Email:fxo853@bham.ac.uk
}

\section{Refereed Paper}

Accepted 19 April 2012

DOI: 10.1038/sj.bdj.2012.667

${ }^{\circledR}$ British Dental Journal 2012; 213: 123-124
Diagnosis was of a non-retentive, unsupportive mandibular distal extension RPD, with bone resorption leading to a prominent mylohyoid ridge resulting in trauma to the overlying mucosa. Moderate chronic generalised periodontitis exacerbated by chronic smoking was also present.

\section{TREATMENT}

Provision of a new acrylic RPD with a different design to the patient's former RPD was first undertaken but this was unsuccessful. The necessary $3 \mathrm{~mm}$ thickness of acrylic connector was incompatible with the tongue due to the amount of spread that had occurred. Any movement of the tongue also caused displacement of the denture. The option of using titanium alloy as the RPD framework was then explored after a patch test revealed no allergy of the patient to titanium alloy.

The conventional clinical and design stages were followed and the resulting framework was well adapted to the ridge, with excellent retention, support and stability (Fig. 1).

\section{DISCUSSION}

This case study combines a patient with a metal allergy and a difficult lower distal extension denture. The impact of metal allergy in practice has received limited clinical report despite the fact that all dental cast alloys release ions into the oral environment and thus, have the potential to interact with the tissues. ${ }^{1}$ Allergy to nickel, gold, cobalt and palladium are the most commonly stated. ${ }^{1}$ Titanium has been

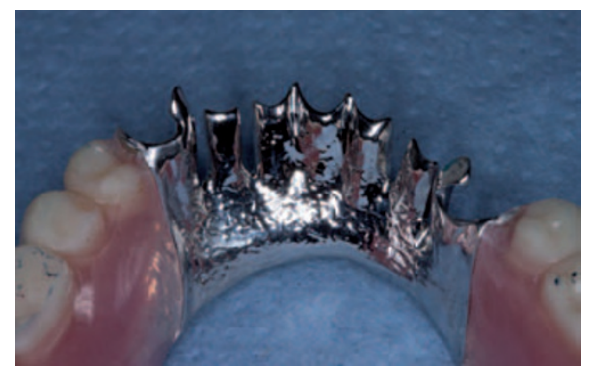

Fig. 1 The titanium casting, which shows similar appearance to traditional metal designs

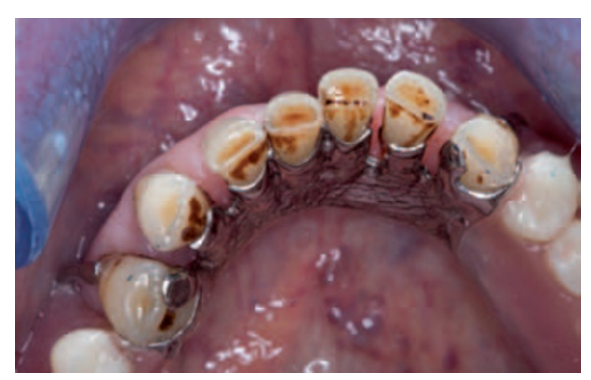

Fig. 2 The titanium casting placed clinically. In spite of the dental staining as a result of smoking and use of chlorhexidine mouthrinse, the casting shows good adaptation and was comfortable for the patient

well identified as an inert, biocompatible metal but its use in removable prostheses is relatively limited, due to casting difficulties, ${ }^{2}$ and lack of clinical studies.

The use of conventional casting alloys such as cobalt chrome was not undertaken due to patch testing revealing allergy to chromium and other metals.

Success of titanium in implantology has been widely proven although titanium implant allergy is likely to become more commonly reported as its use increases. ${ }^{3}$ However, as a partial denture framework it has the advantages of better accuracy 
of fit, light weight, increased patient comfort, high strength and excellent biocompatibility. ${ }^{3}$ The biocompatibility of the alloy is its most important feature in metal allergy cases. Sensitivity reactions to metal only occur when a given metal releases ions into the environment as a result of corrosion. Titanium is known to be highly corrosion resistant due to its ability to form thermodynamically stable and adherent oxide layers on its surface. ${ }^{4}$ The better accuracy of fit stems from the material's ability to be formed into thin plates but still maintain high strength; ${ }^{3}$ this is reflected in the patient's comments about the framework being tight, comfortable and the perfect fit for her mouth. The patient also commented on how light the denture felt which is due to the low density and therefore light weight of the alloy when cast (Fig. 2). However, the low density of the alloy presents a significant problem to the casting process, as does the high melting point. The melting point of titanium is $1,700{ }^{\circ} \mathrm{C}$ resulting in a long burn-out process and porosities appearing in the cast alloy if the alloy is insufficiently melted or inadequate pressure is used. ${ }^{3}$ Radiographic examination of the framework will help determine the presence of porosities, ${ }^{4}$ although these have not been reported as a factor in causing fracture of the titanium framework. ${ }^{2}$ Titanium clasps must also be designed and planned well, as the alloy is more flexible than cobalt-chrome ( $\mathrm{CoCr}$ ), to prevent permanent deformation. ${ }^{3}$ The clasps functioned well in the patient's mouth; they provided adequate retention to prevent displacement of the denture and exhibited good elastic recovery. Economically, the high cost of a titanium framework was once a disadvantage to the use of the metal when compared with conventional metals. However, liaison with laboratory technicians will reveal that this is no longer the case as titanium alloy now has a similar cost to that of cobalt chrome. Specific maintenance instructions on how to care for the denture are required as titanium frameworks have been reported to show surface discolouration with the use of strong alkaline denture cleansers. ${ }^{3}$ Therefore, their use is best avoided. Plaque has also been shown to adhere more easily to titanium alloy than conventional denture alloys so it is important to inform the patient that good oral hygiene standards must be kept. ${ }^{3}$ The patient was encouraged to keep up with her denture hygiene regime of soaking in water overnight but was encouraged to additionally brush the denture after meals.

\section{CONCLUSION}

Titanium alloys are now becoming an acceptable material for the construction of RPDs. Their main disadvantage is cost, although this is likely to change as their popularity increases. The use of titanium is ideal in patients who report a history of allergies to conventional metal alloys used for casting denture frameworks.

1. Schmalz G, Garhammer P. Biological interactions of dental cast alloys with oral tissues. Dent Mater 2002; 18: 396-406.

2. Takayama Y, Takishin N, Tsuchida F, Hosoi T. Survey on use of titanium dentures in Tsurumi University Dental Hospital for 11 years. J Prosthodont Res 2009; 53: 53-59.

3. Ohkubo C, Hanatani S, Hosoi T. Present status of titanium removable dentures - a review of the literature. J Oral Rehabil 2008; 35: 706-714.

4. Könönen $M$, Rintanen J, Waltimo $A$, Kempainen $P$. Titanium framework removable partial denture used for patient allergic to other metals: a clinical report and literature review. J Prosthet Dent 1995; 73: 4-7. 